\title{
Complications of supra-annular mitral valve placement in infants
}

\author{
C L Barker, P E F Daubeney, E A Shinebourne
}

Heart 2005;91:e48 (http://www.heartinl.com/cgi/content/full/91/6/e48). doi: 10.1136/hrt.2004.057877

Two infants underwent supra-annular placement of prosthetic mitral valves. The objective of this strategy was to insert a larger valve and delay replacement. This approach was initially successful but by two and three years later the patients developed impairment of cardiac function. The prosthesis decreased the volume and compliance of the left atrium causing high left atrial and pulmonary venous pressures. The "ventricularised" atrium below the prosthesis dilated. In neither case was it possible to delay second valve replacement.

M itral valve replacement in children is rarely required in the developed world. The native valve annulus in young children often accepts only a small prosthesis. This allows for only a short period of growth before replacement is necessary. Supra-annular positioning of larger prosthetic mitral valves was intended to extend this time for growth. However, we report two cases in which the growth period was not significantly extended and the children became haemodynamically compromised before their second valve replacement.

\section{CASE REPORT}

Patient 1 presented at 16 weeks of age in cardiac failure caused by mitral valve stenosis with a mitral valve annulus of $12 \mathrm{~mm}$. Surgical valvotomy was attempted but the valve remained stenotic. The native valve and papillary muscles were excised and a $19 \mathrm{~mm}$ St Jude prosthesis (St Jude Medical Inc, St Paul, Minnesota, USA) was positioned above the orifice of the left atrial appendage. At 2 years of age she presented with fatigue and dyspnoea and with signs of heart failure. Chest radiography confirmed pulmonary venous congestion. Cross sectional echocardiography showed a dilated left ventricle and a dilated "ventricularised" atrial segment with paradoxical movement outwards during ventricular systole. She had pulmonary hypertension (tricuspid regurgitation with a peak instantaneous Doppler derived pressure difference of $50 \mathrm{~mm} \mathrm{Hg}$ ). She was treated with diuretics with clinical improvement and underwent cardiac catheterisation (fig 1). The mean pulmonary capillary wedge pressure was $17 \mathrm{~mm} \mathrm{Hg}$ and left ventricular end diastolic pressure was $17 \mathrm{~mm} \mathrm{Hg}$. When she was 2 years 6 months old a $21 \mathrm{~mm}$ St Jude prosthesis was successfully positioned in the native valve annulus. Initially her left ventricular function was poor requiring intravenous inotropic support and afterload reduction. Over the next year her cardiac function and left ventricular dimensions normalised.

Patient 2 presented asymptomatically at 12 months with a murmur. Echocardiography confirmed a partial atrioventricular (AV) septal defect with mild left AV valve regurgitation. At 18 months he underwent surgical repair of the AV septal defect. Over the next 12 months his valvar regurgitation worsened and repair was attempted. This was unsuccessful, severe regurgitation remained, and it was not possible to wean him off ventilatory support. Five days later the left AV valve and chordae were excised and a $23 \mathrm{~mm}$ St Jude prosthesis was placed high within the left atrial cavity. He rapidly improved and was extubated within two days. Three years later he presented in pulmonary oedema compounded by atrial flutter. His left ventricular function was impaired and left ventricular end diastolic dimension was $7.1 \mathrm{~cm}$. After he was stabilised with amiodarone and inotropes, cardiac catheterisation showed increased pulmonary capillary wedge pressures. Emergency surgery was required to remove the high atrial prosthesis and to position a $31 \mathrm{~mm}$ St Jude prosthesis in the native left AV valve annulus. Subsequently his left ventricular dimensions normalised and he remains asymptomatic.

\section{DISCUSSION}

In mitral valve stenosis and regurgitation the native valve should be repaired where possible. ${ }^{1}$ Mitral valve replacement in children is infrequently necessary and causes a relatively high mortality, ${ }^{1}$ although this is reducing with greater experience. ${ }^{2}$ Replacement is particularly difficult if the valve annulus is small. Age $<2$ years, low weight and prosthesis size $<20 \mathrm{~mm}$ put children into a high risk group for requirement of a second valve replacement. ${ }^{3}$ Placement of a larger valve into the native annulus is not the answer because a high prosthetic valve size to patient weight ratio is a

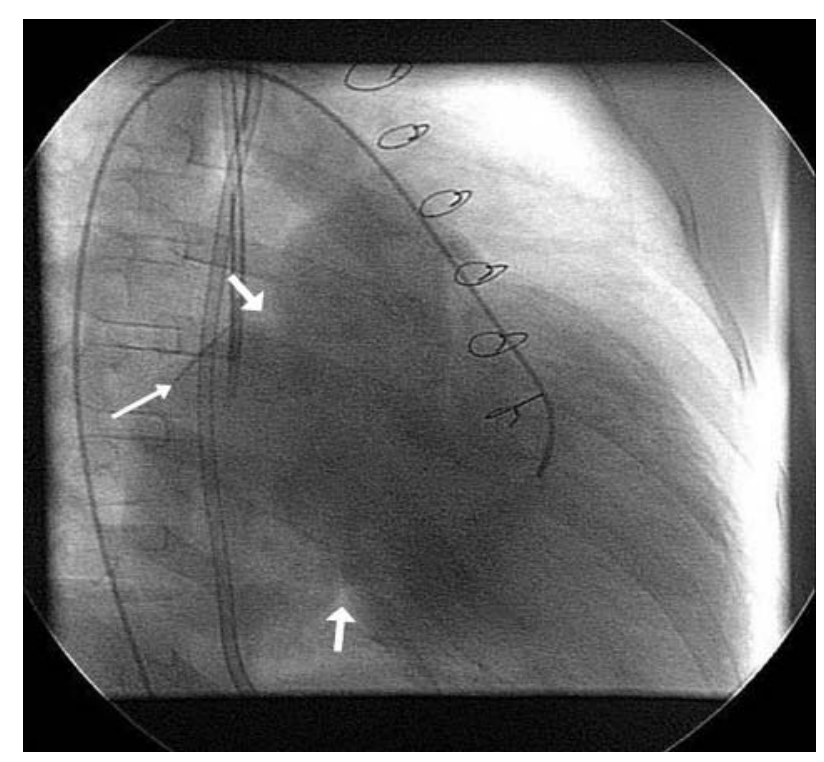

Figure 1 Case 1. Cardiac catheterisation showing supra-annular mitral valve (narrow arrow) and dilated left atrium above the native orifice (wide arrows). 
predictor for early mortality. ${ }^{4}$ The large prosthesis may obstruct the left ventricular outflow tract .It may impinge on the conducting system, and damage to the circumflex coronary artery has been reported. ${ }^{1}$ In placing a larger valve into the already dilated left atrium we had hoped to delay a second replacement.

There are disadvantages to supra-annular placement of the mitral valve. The prosthesis decreases the volume and compliance of the left atrium causing high left atrial and pulmonary venous pressures. The relatively compliant atrium below the prosthesis becomes dilated and its paradoxical movement during ventricular systole acts as an energy sump.

These children managed only two and three years until second valve replacement. Both had left ventricular failure at the time of repeat operation. We feel that in placing a supraannular valve the time to a second replacement was not significantly extended and that major haemodynamic compromise occurred. On the basis of our experience we do not recommend this strategy. With the availability of smaller mechanical prosthetic valves it is safer to position the initial device in the native annulus.
Authors' affiliations

C L Barker, P E F Daubeney, E A Shinebourne, Royal Brompton Hospital, London, UK

Correspondence to: Dr Claire L Barker, Royal Brompton Hospital, Sydney Street, London SW3 6NP, UK; cbarker@doctors.org.uk

Accepted 13 January 2005

\section{REFERENCES}

1 Van Doorn C, Yates R, Tsang V, et al. Mitral valve replacement in children: mortality, morbidity, and haemodynamic status up to medium term follow up. Heart 2000;84:636-42.

2 Alexiou C, Galogavrou M, Chen Q, et al. Mitral valve replacement with mechanical prostheses in children: improved operative risk and survival. Eur J Cardiothorac Surg 2001:20:105-13.

3 Raghuveer G, Caldarone C, Hills C, et al. Predictors of prosthesis survival, growth, and functional status following mechanical mitral valve replacement in children aged $<5$ years: a multi-institutional study. Circulation 2003;108(suppl 2):174-9.

4 Caldarone C, Raghuveer G, Hills $C$, et al. Long-term survival after mitral valve replacement in children aged $<5$ years: a multi-institutional study. Circulation 2001;104(suppl 1):143-7. 